\title{
Closed-Loop Acoustic Stimulation During Sedation with Dexmedetomidine (CLASS-D): Protocol for a Within-Subject, Crossover, Controlled, Interventional Trial with Healthy Volunteers
}

This article was published in the following Dove Press journal:

Nature and Science of Sleep

\author{
Christian S Guay $\mathbb{D}^{\prime}$ \\ Alyssa K Labonte' \\ Michael C Montana' \\ Eric C Landsness (D) $^{2}$ \\ Brendan P Lucey (D) ${ }^{2}$ \\ MohammadMehdi Kafashan' \\ Simon Haroutounian' \\ Michael S Avidan ${ }^{1,3}$ \\ Emery N Brown ${ }^{4,5}$ \\ Ben Julian A Palanca $\mathbb{D}^{1,3,6,7}$ \\ 'Department of Anesthesiology, \\ Washington University School of Medicine \\ in St. Louis, St. Louis, MO, USA; \\ ${ }^{2}$ Department of Neurology, Division of \\ Sleep Medicine, Washington University \\ School of Medicine in St. Louis, St. Louis, \\ MO, USA; ${ }^{3}$ Department of Psychiatry, \\ Washington University School of Medicine \\ in St. Louis, St. Louis, MO, USA; \\ ${ }^{4}$ Department of Brain and Cognitive \\ Sciences, Massachusetts Institute of \\ Technology, Cambridge, MA, USA; \\ ${ }^{5}$ Department of Anesthesia, Critical Care \\ and Pain Medicine, Massachusetts General \\ Hospital, Harvard Medical School, Boston, \\ MA, USA; ${ }^{6}$ Division of Biology and \\ Biomedical Sciences, Washington \\ University School of Medicine in St. Louis, \\ St. Louis, MO, USA; ${ }^{7}$ Department of \\ Biomedical Engineering, Washington \\ University in St. Louis, St. Louis, MO, USA
}

Correspondence: Ben Julian A Palanca Washington University School of Medicine in St. Louis, Department of Anesthesiology, 660 S. Euclid Avenue, Box 8054, St. Louis, MO, 63110, USA

Tel + I 3|4-273-9076

Fax + I 3|4-747-3977

Email palancab@wustl.edu
Introduction: The relative power of slow-delta oscillations in the electroencephalogram (EEG), termed slow-wave activity (SWA), correlates with level of unconsciousness. Acoustic enhancement of SWA has been reported for sleep states, but it remains unknown if pharmacologically induced SWA can be enhanced using sound. Dexmedetomidine is a sedative whose EEG oscillations resemble those of natural sleep. This pilot study was designed to investigate whether SWA can be enhanced using closed-loop acoustic stimulation during sedation (CLASS) with dexmedetomidine.

Methods: Closed-Loop Acoustic Stimulation during Sedation with Dexmedetomidine (CLASS-D) is a within-subject, crossover, controlled, interventional trial with healthy volunteers. Each participant will be sedated with a dexmedetomidine target-controlled infusion (TCI). Participants will undergo three CLASS conditions in a multiple crossover design: in-phase (phase-locked to slow-wave upslopes), anti-phase (phase-locked to slow-wave downslopes) and sham (silence). High-density EEG recordings will assess the effects of CLASS across the scalp. A volitional behavioral task and sequential thermal arousals will assess the anesthetic effects of CLASS. Ambulatory sleep studies will be performed on nights immediately preceding and following the sedation session. EEG effects of CLASS will be assessed using linear mixedeffects models. The impacts of CLASS on behavior and arousal thresholds will be assessed using logistic regression modeling. Parametric modeling will determine differences in sleepiness and measures of sleep homeostasis before and after sedation.

Results: The primary outcome of this pilot study is the effect of CLASS on EEG slow waves. Secondary outcomes include the effects of CLASS on the following: performance of a volitional task, arousal thresholds, and subsequent sleep.

Discussion: This investigation will elucidate 1) the potential of exogenous sensory stimulation to potentiate SWA during sedation; 2) the physiologic significance of this intervention; and 3) the connection between EEG slow-waves observed during sleep and sedation.

Keywords: sleep, anesthesia, consciousness, dexmedetomidine, electroencephalography, acoustic stimulation

\section{Introduction}

\section{Enhancement of EEG Slow Oscillations During Sleep}

Sleep addresses critical physiologic needs in humans and most living organisms. Although the neurological mechanisms underlying sleep are still under investigation, the electroencephalographic (EEG) correlates of sleep states are 
established. ${ }^{1}$ Slow $(0.1-1 \mathrm{~Hz})$ and delta $(1-4 \mathrm{~Hz})$ waves are large amplitude, low-frequency EEG oscillations that define non-rapid eye movement (NREM) sleep stage 3 (N3). Importantly, they have been shown to play roles in physiologic restoration, memory consolidation and dissipating homeostatic sleep pressure. ${ }^{2}$ At the cellular level, they correspond to the synchronous state switching of large cortical neuron ensembles between depolarized "up" states and hyperpolarized "down" states (ie, burst firing). ${ }^{3}$ At the scalp sensor level, these oscillations are traditionally quantified through slowwave activity (SWA)-EEG relative power in the $0.5-4 \mathrm{~Hz}$ frequency band. ${ }^{4} \mathrm{SWA}$ is a known marker of sleep need, and its dissipation is homeostatically regulated. ${ }^{5}$

Enhancement of SWA has recently emerged as a promising strategy to improve natural sleep and treat sleep disorders using electrical, magnetic and acoustic modalities. ${ }^{6-8}$ The impact of these interventions on clinical outcomes is an active area of investigation. Preliminary findings have raised the possibility of accelerating the decay of sleep pressure, enhancing memory consolidation and improving autonomic, neuroendocrine and immune function. ${ }^{9-14}$ Considering the substantial safety advantages of using sound over other modalities, acoustic stimulation has become the preferred method to enhance sleep slow oscillations in the ambulatory setting. ${ }^{9,15}$ This approach depends on the real-time processing of EEG signals and on the precise delivery of auditory stimulation in relation to the phase of oscillations. Over the last decade, multiple algorithms have been developed to synchronize bursts of pink noise inphase with the upslope of slow oscillations. Pink noise, also known as $1 / \mathrm{f}$ noise (denoting its power density), is random noise that has equal energy per octave. Acoustic stimuli with this spectral distribution are hypothesized to transduce through the vestibulocochlear nerve and ascending non-lemniscal pathway, with diffuse recruitment of cortical neurons. Synchronized depolarization cycles follow a slow oscillatory profile reflected by subsequent augmentation in SWA in the scalp EEG. ${ }^{16}$ Conversely, anti-phase stimulation (i.e., acoustic stimuli synchronized with the downslope of slow-waves) has been shown to be disruptive, as inferred by decrements in SWA. ${ }^{8}$ Thus, precisely timed auditory stimulation can either potentiate or undermine the manifestation of EEG slow-wave oscillations.

\section{Enhancement of EEG Slow Oscillations During Sedation}

Among sedatives used in clinical practice, dexmedetomidine is posited to induce states most akin to N3 sleep. ${ }^{17} \mathrm{An}$ adrenergic alpha-2 agonist, dexmedetomidine primarily induces slow-waves and sedation by inhibiting the release of norepinephrine from the locus coeruleus, thus allowing the ventrolateral preoptic nucleus to inhibit ascending arousal circuits. ${ }^{18}$ This is in contrast to the majority of anesthetics that induce slow-waves by potentiating the gamma aminobutyric acid (GABA)-A receptor. ${ }^{19}$ The EEG scalp topography of dexmedetomidine sedation has been described using high-density recordings; ${ }^{20,21}$ however, cortical and subcortical source localization have not yet been undertaken. At sufficient doses, the behavioral phenotype of dexmedetomidine sedation resembles that of NREM sleep, characterized by intact spontaneous respirations, hemodynamic stability and susceptibility to arousal. $^{22,23}$ These properties have made dexmedetomidine a popular sedative in the critical care setting. As with natural sleep, SWA correlates with level of unconsciousness during sedation. ${ }^{23,24}$ Nonetheless, important mechanistic differences between dexmedetomidine sedation and natural sleep exist, and their relationship remains an area of active investigation. ${ }^{25}$

Despite a rapidly growing literature describing acoustic enhancement of slow oscillations during natural NREM sleep, the effects of closed-loop acoustic stimulation during sedation (CLASS) remain unknown. From a scientific perspective, differential effects of acoustic stimulation during sleep and sedation may shed light on distinct underlying mechanisms and the relationships between these states. Moreover, for EEG slow-waves occurring during dexmedetomidine sedation, estimation of sources and effects on sleep homeostasis remain poorly characterized. From a clinical perspective, non-pharmacologic enhancement of slow oscillations during sedation would have important implications. Considering the relationship of SWA with level of unconsciousness, ${ }^{26}$ it may be feasible to use lower drug doses to achieve similar brain states and behavioral phenotypes. This would be particularly useful when deeper states of sedation are needed, infusion rates are limited by side effects (eg, bradycardia or respiratory depression), and poor physiologic reserve or hemodynamic instability limit dose escalation. Improvements in sleep quality and dissipation of sleep pressure using dexmedetomidine and phase-locked neurostimulation may 
also have positive effects on clinical outcomes in intensive care units. Further, reduced exposure to anesthetic agents through neurostimulation may reduce the incidence and severity of post-operative complications and neurocognitive disorders. To this end, a recent randomized controlled trial described the use of low frequency auditory binaural beats and visual stimulation to decrease the cumulative propofol dose administered to pediatric patients during sedation for sub-umbilical surgery. ${ }^{27}$ While there were no significant effects on the Bispectral Index, a proprietary and unitless processed EEG measure utilized for monitoring intraoperative "anesthetic depth," quantitative analysis of the EEG was not undertaken.,28,29

In summary, closed-loop acoustic stimulation (CLAS) can enhance EEG slow oscillations during natural sleep, but whether auditory stimuli can enhance slow oscillations during sedation is unknown. Acoustic enhancement of slow oscillations could provide clinicians with a nonpharmacological adjunct to augment thresholds for arousal, with potential translation toward improved clinical outcomes in the perioperative and critical care settings.

\section{Objectives and Specific Aims}

The primary objective of this study is to determine if phaselocked acoustic stimulation can enhance EEG slow-wave expression during sedation with dexmedetomidine. Secondary objectives are to characterize dexmedetomidine slow-waves with and without CLASS using high-density EEG, and to measure their downstream effects on behavior, arousal, and sleep. The corresponding specific aims are as follows:

\section{Specific Aim I}

Determine whether dexmedetomidine-induced EEG slowwaves can be potentiated with CLASS.

\section{Specific Aim 2}

Assess whether performance of a behavioral task during dexmedetomidine sedation is modulated by CLASS.

\section{Specific Aim 3}

Assess whether thresholds for responsiveness to peripheral thermal stimulation during dexmedetomidine sedation are modulated by CLASS.

\section{Specific Aim 4}

Assess whether CLASS with dexmedetomidine reduces metrics of slow-wave need on the night of the intervention.

\section{Specific Aim 5}

Compare modeled source localization of dexmedetomidine-induced EEG slow-waves recorded in the presence and absence of CLASS.

\section{Methods and Analysis Study Design}

Closed-Loop Acoustic Stimulation during Sedation with Dexmedetomidine (CLASS-D) is a prospective, withinsubject, crossover, controlled, interventional pilot trial (ClinicalTrials.gov NCT4206059). For each participant, study participation will focus on a single session of dexmedetomidine sedation flanked by ambulatory sleep studies targeting pre- and post-sedation sleep structure (Figure 1). The study design will allow investigators to compare the effects of three acoustic stimulation conditions on EEG slowwaves in the same brain, in close temporal proximity, and at the same plasma concentration of dexmedetomidine. The three conditions are: in-phase stimulation (pink noise bursts synchronized to the upslope of slow-waves), anti-phase stimulation (pink noise bursts synchronized to the downslope of slow-waves), and sham stimulation (volume set to zero $\mathrm{dB}$ ) (Figure 2). Washout periods of acoustic silence will be used during the stimulation protocol to control for short-term resonant effects of acoustic stimulation. The rate of dexmedetomidine infusion will be adjusted throughout the experiment to maintain stable plasma concentrations, with post hoc assessment of plasma levels. Participants will be compensated 20.00 USD for the home sleep studies, 180.00 USD for the sedation session and 50.00 USD for an elective noncontrast brain MRI.

\section{Study Procedures and Data Acquisition Recruitment and Baseline Testing}

Participants will be recruited using posted fliers, internet advertisements and university volunteer databases. Prior to enrollment, participants will undergo a phone interview to screen for inclusion and exclusion criteria (Table 1).

Days prior to the planned sedation session, participants will present to Washington University Medical Center for the first stage of study protocol. At this visit, informed consent will be obtained, and each participant will complete a battery of questionnaires to assess his/her overall health and sleep quality.

\section{Quantitative Sensory Testing}

Each participant will then undergo training on quantitative sensory testing (QST). Baseline heat pain threshold (HPT) 


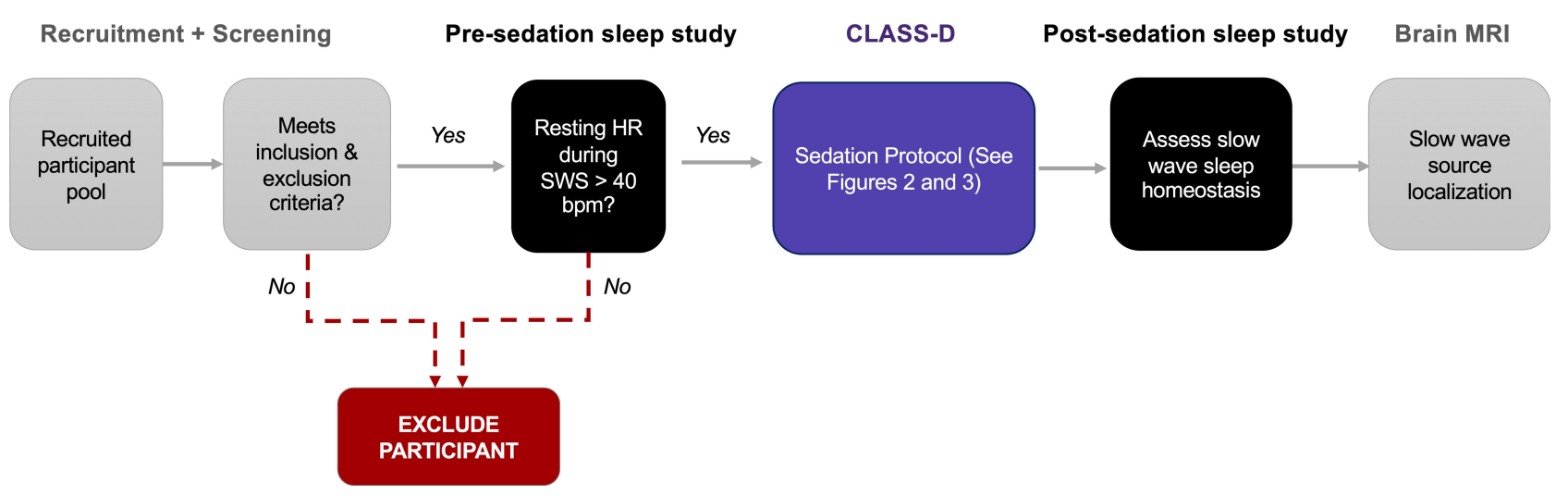

Figure I Longitudinal study design. After being recruited, participants will be evaluated at the medical center. If they meet inclusion and exclusion criteria, they will be trained on how to use the Dreem device and record sleep EEG data prior to undergoing sedation. Participants will also record sleep EEG data on nights following sedation. A non-contrast structural brain magnetic resonance imaging (MRI) scan will then be completed at a later date.

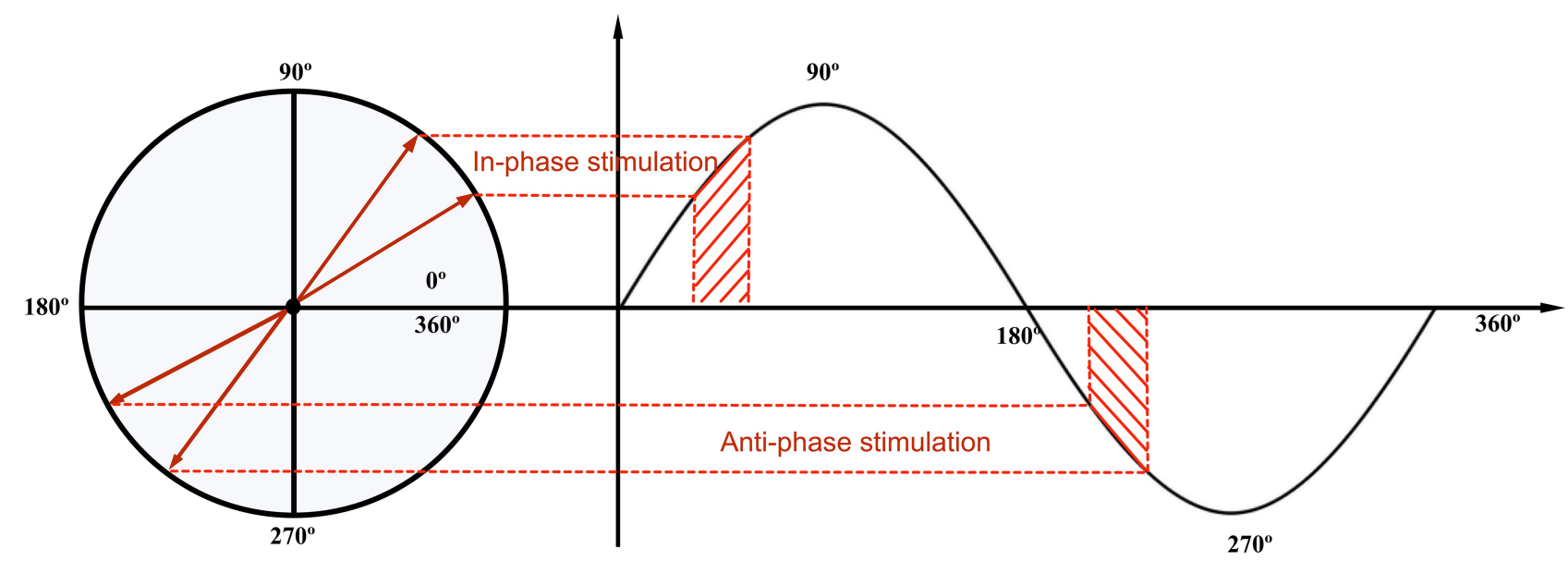

Figure 2 Phase-locked acoustic stimulation. A circular plot can be used to visualize different phases of slow-waves that are targeted using phase-locked acoustic stimulation. In-phase stimulation delivers pink noise during the upslope of slow-waves, and anti-phase stimulation delivers pink noise during the downslope of slow-waves. In practice, acoustic stimulation is phase-locked to a range of the slow-wave's cycle, represented in red here.

and heat pain tolerance threshold (HPTT) will be measured with a Thermal Sensory Analyzer (TSA-II, Medoc, Israel), using the method of limits. ${ }^{30}$ Persistently elevated

Table I Inclusion and Exclusion Criteria for CLASS-D

\begin{tabular}{|c|c|}
\hline Inclusion Criteria & Exclusion Criteria \\
\hline $\begin{array}{l}\text { - } 18-40 \text { years old } \\
\text { - ASA Physical Status I }-2\end{array}$ & $\begin{array}{l}\text { - Diagnosed sleep disorders } \\
\text { - Habitually short sleeper } \\
\text { - Use of psychoactive medication } \\
\text { - Diagnosed psychiatric disorder } \\
\text { - Diagnosed hearing disorder } \\
\text { - Neck circumference }>40 \mathrm{~cm} \\
\text { - BMI >30 } \\
\text { - Recreational drug or nicotine use } \\
\text { - Pregnant or nursing female } \\
\text { - Resting HR }<40 \text { bpm during SWS } \\
\text { - QST pain threshold }>50^{\circ} \mathrm{C}\end{array}$ \\
\hline
\end{tabular}

Note: Participants will be screened for inclusion and exclusion criteria at the original recruitment interview and on the day of sedation.
HPTT $>50^{\circ} \mathrm{C}$ or inability to provide consistent QST responses will lead to exclusion from the study. Please refer to Supplemental Methods for additional details.

\section{Ambulatory Sleep Recording}

Each participant will then be provided with a Dreem device, designed to record sleep in the home environment and to deliver in-phase acoustic stimulation via bone conduction. $^{15,31,32}$ The device includes frontal and occipital EEG, as well as pulse plethysmography and accelerometry. Participants will receive instructions on using the device to record ambulatory sleep studies. On nights prior to sedation, each participant will wear the device and physiological data will be recorded (ie, pre-sedation sleep). The acoustic stimulation functions of the device will be enabled for one of the pre-sedation nights. To assess the effect of CLASS and dexmedetomidine sedation on subsequent nocturnal sleep architecture, participants 
will wear the device on the night following sedation (postsedation sleep). Participants will receive standard presedation fasting instructions (nil by mouth for 2 hours for clears, 4 hours for liquids, 6 hours for meals) for the dexmedetomidine sedation session.

\section{Pre-Sedation Procedures}

A target-controlled infusion (TCI) of dexmedetomidine based on validated pharmacokinetic models will be used to achieve stable plasma concentrations during this study. $^{23,24,33-35}$ The target-controlled infusion syringe pump is controlled by Rugloop IIC, a Windows ${ }^{\circledR}$ based target-controlled infusion and data management program. Prior to each sedation session, Rugloop II will be used to simulate the sedation session for each possible targeted concentration (ie, 2, 3, and $4 \mathrm{ng} / \mathrm{mL}$ ). The individualized drug administration simulation will be reviewed by a board-certified anesthesiologist prior to the sedation session. For use in the critical care setting, the manufacturer's label suggests a dexmedetomidine loading dose regimen of $6 \mathrm{mcg} / \mathrm{kg} / \mathrm{hr}$ for 10 minutes, followed by lower maintenance infusion rates. ${ }^{36}$ The default maximal infusion rate for dexmedetomidine in Rugloop II is $6 \mathrm{mcg} / \mathrm{kg} / \mathrm{hr}$, consistent with previous volunteer studies. ${ }^{20,33,34}$ These precautions are primarily in place to minimize the risk of hypotension and bradycardia. Phenylephrine and glycopyrrolate will be used to treat hemodynamic effects of dexmedetomidine at the discretion of the attending anesthesiologist.

On the day of sedation, the research team will readminister the screening questionnaire to ensure that the participant still meets the inclusion/exclusion criteria. The ambulatory sleep study will be reviewed. Staff will ensure that the participant has adhered to American Society of Anesthesiologists (ASA) fasting guidelines for sedation/ general anesthesia. Pre-sedation sleepiness will be assessed using the Stanford Sleepiness Scale. ${ }^{37}$

The sedation protocol will be completed in a location equipped with standard monitoring, airway equipment, suction, and a dedicated board-certified anesthesiologist, per standard of practice guidelines for anesthesia care. ${ }^{38}$ Each participant will have two intravenous catheters (IV) placed, one to receive medications and fluid throughout the study protocol, and another to draw blood samples. Standard ASA monitors will be applied: electrocardiogram, non-invasive blood pressure, pulse oximeter, and breathing rate monitors. Participants will also have a highdensity 65-electrode EEG scalp electrode net (MagStim) applied with Elefix conductive gel (Nihon Kohden) injected within sensors. Additionally, three shielded electrodes will be affixed to the scalp and connected to the Elemind device (Elemind Technologies, Inc., Cambridge, MA): one active electrode, one reference electrode, and one ground electrode. The Elemind device consists of EEG amplifiers, a circuit board, a sound card, a visual display, and a battery, all shielded in a metal case and connected to a separate computer for software interfacing running custom Labview software described below. The device amplifies EEG input, tracks the phase of a specified central frequency in the band-passed signal, and synchronizes audio output to the phase specified by the investigators.

Following application of all monitors, a QST will be performed to measure HPT and HPTT at the non-dominant forearm site used in previous testing. If HPTT is not greater than $50^{\circ} \mathrm{C}$, the area will be marked. If HPTT is greater than $50^{\circ} \mathrm{C}$, the test will be repeated at a different location on the non-dominant forearm and repeated until the HPTT is found to be less than $50^{\circ} \mathrm{C}$. The final location will then be marked, and all subsequent QST measurements will be made at this location. EEG data will be recorded during HPPT testing.

Participants will be instructed to perform a validated behavioral task ${ }^{39,40}$ throughout the protocol by complying with the following instruction during eye closure: "Squeeze the dynamometer during inspiration and release it during expiration". Participants will be considered responsive whenever they perform the task consistently (at least five consecutive correct squeezes), and unresponsive after five consecutive failures to squeeze during inspiration. State-space models will also be used to assess loss and return of responsiveness offline. ${ }^{41}$ After confirming adequate comprehension, 10 minutes of baseline awake EEG measurements will be recorded: five minutes with eyes open and five minutes with eyes closed. After completing all questionnaires and baseline recordings, and ensuring that all monitors are functional, the sedation protocol will begin (Figure 3). The protocol can be broadly separated into six phases: Phase I, light dexmedetomidine titration; Phase II, light CLASS; Phase III, deep dexmedetomidine titration; Phase IV, deep CLASS; Phase V, arousal; Phase VI, recovery. All phases will be recorded using a video camera. Table 2 outlines the alignment of experimental phases and study specific aims. 


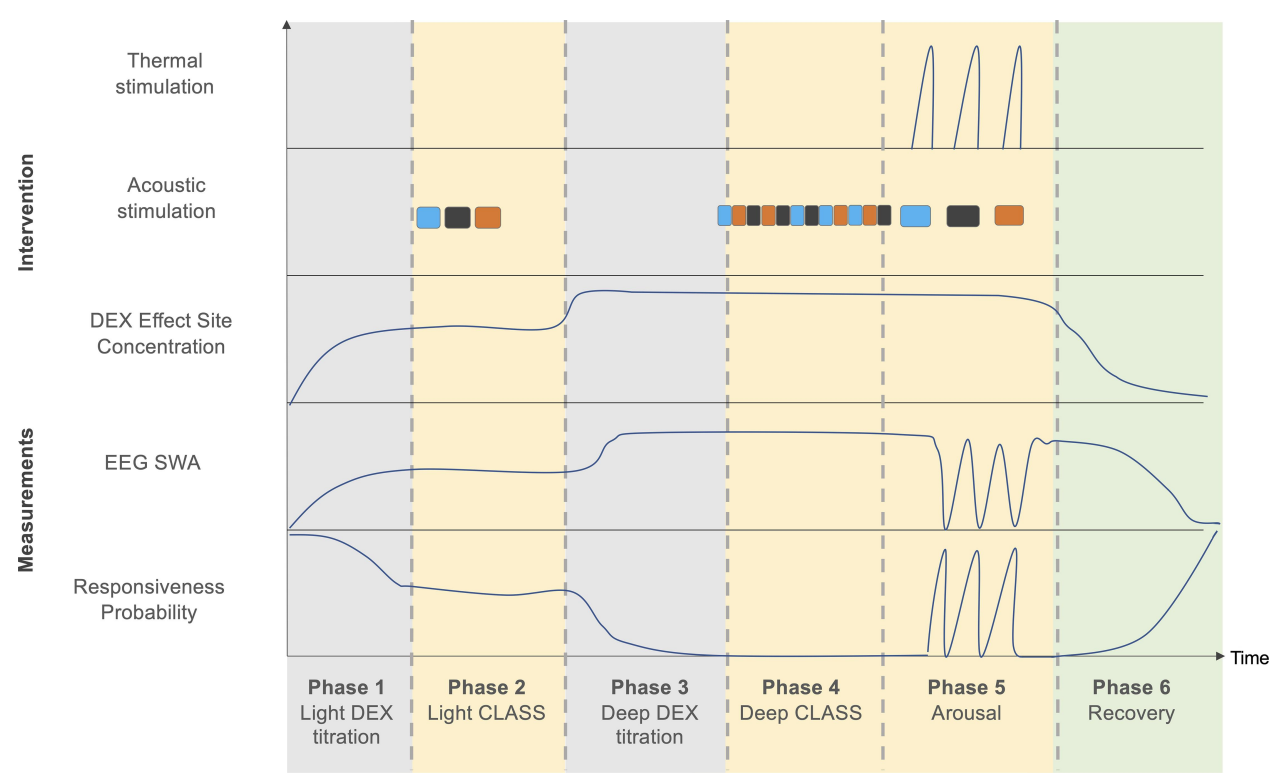

Figure 3 Sedation protocol. The sedation protocol consists of six phases. In Phase I, dexmedetomidine will be escalated using TCl, causing a subsequent increase in SWA and impairments in the behavioral task. During Phase II, responsive participants showing EEG slow-waves will be presented with CLASS conditions while they are still performing the behavioral task. Upon entry into Phase III, dexmedetomidine will be further escalated to achieve loss of behavioral responsiveness. Phase IV represents the core of the experiment. Participants unresponsive and demonstrating EEG slow-waves will be presented with multiple blocks of CLASS conditions during a stable dexmedetomidine effect site concentration. Targeting of this dexmedetomidine concentration will be continued during Phase V. Participants will be aroused three times by thermal stimulation during CLASS. In Phase VI, the dexmedetomidine infusion will stop and resting EEG will be recorded as participants recover full responsiveness during the behavioral task. Blue, black and orange squares represent in-phase, antiphase and sham conditions, respectively. The order of CLASS conditions will be randomized across participants.

\section{Phase I: Light Dexmedetomidine Titration}

The participant will be instructed to close his/her eyes and perform the behavioral instruction: "Squeeze the

Table 2 CLASS-D Phases and Aims

\begin{tabular}{|l|l|l|}
\hline Phase \# & \multicolumn{1}{|c|}{ Condition } & \multicolumn{1}{|c|}{ Aims } \\
\hline Phase 0 & $\begin{array}{l}\text { Pre-sedation } \\
\text { sleep study }\end{array}$ & Assess effects of CLASS on sleep (Aim 4) \\
\hline Phase I & $\begin{array}{l}\text { Light DEX } \\
\text { titration }\end{array}$ & Induce EEG slow waves \\
\hline Phase II & Light CLASS & Test effects of CLASS on behavior (Aim 2) \\
\hline Phase III & $\begin{array}{l}\text { Deep DEX } \\
\text { titration }\end{array}$ & $\begin{array}{l}\text { Induce loss of behavioral } \\
\text { responsiveness }\end{array}$ \\
\hline Phase IV & $\begin{array}{l}\text { Deep CLASS } \\
\text { Test effects of CLASS on slow waves } \\
\text { (Aims I and 5) }\end{array}$ \\
\hline Phase V & Arousal & $\begin{array}{l}\text { Test effects of CLASS on arousal } \\
\text { thresholds (Aim 3) }\end{array}$ \\
\hline Phase VI & Recovery & $\begin{array}{l}\text { Drug elimination, transition to waking } \\
\text { state }\end{array}$ \\
\hline Phase VII & $\begin{array}{l}\text { Post-sedation } \\
\text { sleep study }\end{array}$ & Assess effects of CLASS on sleep (Aim 4) \\
\hline
\end{tabular}

Notes: Aims I, 2 and 3 will be directly tested during the sedation protocol. Aim 4 will be completed using the sleep studies on nights preceding and following the sedation protocol. Aim 5 will be addressed by combining analysis of EEG data from the sedation protocol with a structural brain MRI acquired at a later date. dynamometer during inspiration and release it during expiration". An initial target concentration of $2 \mathrm{ng} / \mathrm{mL}$ will be entered into Rugloop and the infusion will begin. Once the predicted plasma site concentrations of dexmedetomidine have reached $2 \mathrm{ng} / \mathrm{mL}$, two criteria will be assessed to determine if the experiment will progress to the light or deep acoustic stimulation phase (Figure 4). The first criterion is behavioral: investigators will assess if the participant is still performing the task of squeezing as device in their dominant hand during inspiration. The second criterion is electrophysiological: investigators will visually assess for the presence of large amplitude ( $>20$ microvolts) EEG slowwaves. The staff assessing the EEG will have completed accredited training in the interpretation of human EEG during sedation and anesthesia. ${ }^{42}$ If the participant demonstrates the presence of EEG slow-waves (Criterion 1) and is still performing the behavioral task (Criterion 2), the experiment will proceed to light CLASS (Phase II). Alternatively, if the participant loses behavioral responsiveness before or concurrent with the appearance of EEG slow-waves, then the experiment will proceed directly to deep CLASS (Phase IV), bypassing Phases II and III.

\section{Phase II: Light CLASS}

To enable phase-locked stimulation, the instantaneous phase of EEG slow oscillations will be computed in real-time by the 


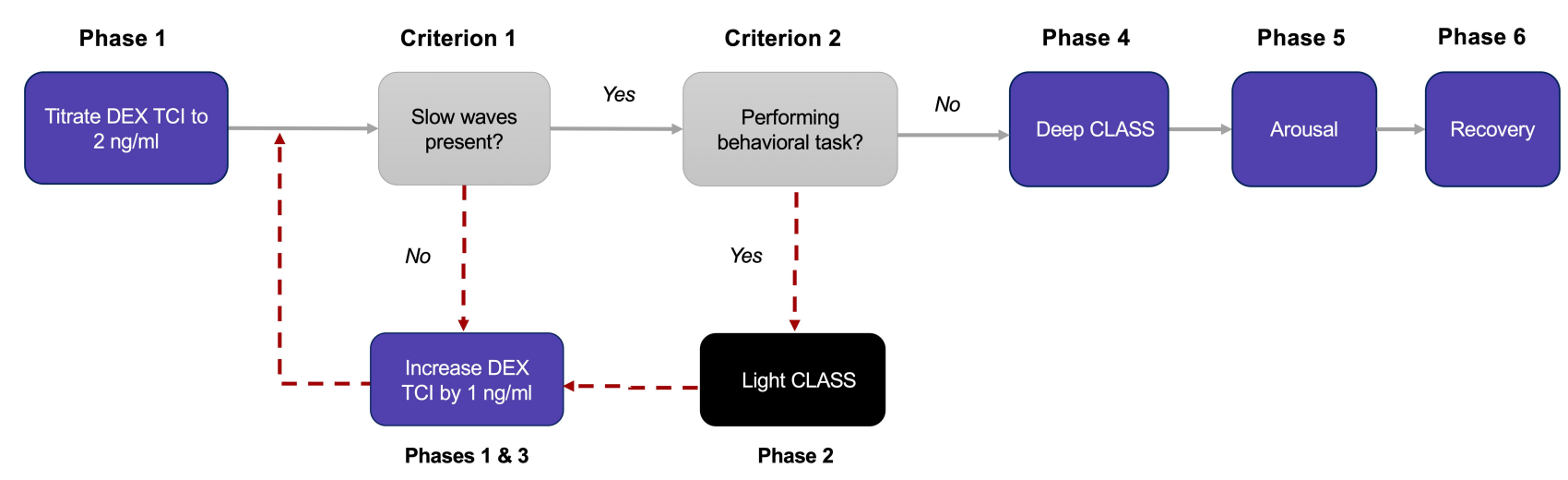

Figure 4 Decision tree during sedation protocol. In all cases, the sedation protocol will begin with the targeting of effect site and plasma dexmedetomidine concentrations of $2 \mathrm{ng} / \mathrm{mL}$ via TCl. In the event that slow-waves are not observed in the EEG, the target concentration will be increased in increments of I ng/mL to induce EEG slow-waves. If a participant with slow-waves continues to perform the behavioral task, they will proceed to light CLASS, after which the dexmedetomidine TCl is increased in increments of I $\mathrm{ng} / \mathrm{mL}$ until they stop performing the task. This is followed by the deep CLASS, arousal, and recovery phases.

Elemind device using the method of endpoint corrected Hilbert transform (ecHT), recently described in detail. ${ }^{14}$ The central frequency for phase-locking will be set to a default of $1 \mathrm{~Hz}$ and modified as needed for each participant to match their dominant slow wave frequency during sedation. Acoustic stimuli will be delivered binaurally via noiseisolating earphones as bursts of pink $1 / \mathrm{f}$ noise of $50 \mathrm{~ms}$ duration with a $5 \mathrm{~ms}$ rising and falling time, identical to those used in the original Ngo study. ${ }^{8}$ Sound volume will be calibrated to $60 \mathrm{~dB}$ using a sound level meter and adjusted for each participant to minimize the risk of arousing them with sound. In contrast to the spontaneous trains of 2-3 grouped slow oscillations observed during slow wave sleep, ${ }^{8,43}$ the slow oscillations observed during dexmedetomidine sedation are more consistent and stable. ${ }^{19,20}$ Therefore, we have shortened the traditional 2.5 second refractory period ${ }^{8}$ to 0.5 seconds, to facilitate continuous stimulation of slow oscillations during CLASS conditions.

Each participant will be presented with the three CLASS conditions as they perform the behavioral task in a sedated state: six one minute blocks of in-phase CLASS, six one minute blocks of anti-phase CLASS, and six one minute blocks of sham (Figure 3). The order of CLASS conditions will be balanced within and across participants to account for lasting resonant effects beyond termination of condition. A one-minute period of silence will also separate conditions as an additional washout period. Assessors of behavioral responses will be blinded to CLASS conditions.

\section{Phase III: Deep Dexmedetomidine Titration}

The dexmedetomidine TCI will be increased in increments of $1 \mathrm{ng} / \mathrm{mL}$ to achieve loss of behavioral responsiveness after which a sample acoustic stimulation train will be delivered to ensure that the participant is not roused by the sound. A conservative estimate for the equilibration constant $\left(t_{1 / 2}\right.$ $\mathrm{k}_{\mathrm{e} 0}$ ) of dexmedetomidine in healthy volunteers is 3 minutes. ${ }^{44}$ Therefore, 15 minutes (ie, $5 \mathrm{x} \mathrm{t}_{1 / 2} \mathrm{k}_{\mathrm{e} 0}$ ) will be allowed to pass before drawing venous samples for quantitation of dexmedetomidine plasma concentration, and beginning Phase IV.

\section{Phase IV: Deep CLASS}

The acoustic stimuli delivered during Phase IV are the same as in Phase II. The volume will be reduced if it arouses the participant. The fundamental differences between phase II and phase IV are threefold: (1) Dexmedetomidine concentrations are higher; (2) Participants are unresponsive; and (3) Each participant is exposed to each CLASS condition four times (Figure 3). The order of the conditions for all participants is predetermined prior to recruitment and balanced across participants to account for carry-over effects. Similar to Phase II, one minute of washout silence will separate each CLASS condition. In participants with enough scalp space, the acoustic stimulation component of the Dreem device will also be tested during deep CLASS.

\section{Phase V: Thermal Arousal}

Participants will undergo three arousals during this phase of the experiment: one during in-phase stimulation, one during anti-phase stimulation, and one during sham stimulation (Figure 2). The order of CLASS conditions under which a participant will be aroused is the same as in Phase II, which is balanced across participants. Each arousal will be preceded by at least three consecutive minutes of acoustic stimulation, which will continue during the arousal procedure. The arousal procedure consists of the same 
ramping thermal stimulation used during pre-sedation QST. The cardinal difference between pre-sedation QST and arousal QST is that one of the investigators will be tasked with pressing the button to end the thermal ramp instead of the sedated participant. The investigator will end the arousal protocol as soon as they observe purposeful behavior in response to the thermal stimulation. The investigator will also be able to terminate the thermal stimulation at their clinical discretion. Vital signs, EEG, and video recording will allow review of the time of arousal during post hoc analysis. EEG changes reflective of arousal during dexmedetomidine sedation consist of decreasing delta and alpha power. ${ }^{23}$ Assessors of arousal will be blinded to the acoustic stimulation conditions. Once a participant has been definitively aroused, thermal stimulation will cease. The participant will then be asked to rate pain on a visual analog scale, and a 15-minute washout period will ensue without any thermal or acoustic stimulation. Based on a recent investigation, ${ }^{23}$ we expect participants to return to a state of unresponsiveness within 60 seconds, and for EEG SWA to return to pre-arousal levels within 10 minutes. This protocol will be repeated three times, once for each stimulation condition. The predicted plasma concentration of dexmedetomidine will be maintained by TCI throughout the acoustic stimulation and arousal phases of the experiment. After the third arousal and washout period, a second $5 \mathrm{~mL}$ venous blood sample will be drawn for quantitation of dexmedetomidine concentration and placed on ice for processing. The dexmedetomidine infusion rate will then be set to zero, and the recovery phase will begin.

\section{Phase VI: Recovery}

All physiological behavioral monitoring will continue as in Phase I. The participant will be monitored until standard discharge criteria are met, including recovery of baseline cognitive and physiologic functions. ${ }^{45} \mathrm{~A}$ post-sedation questionnaire will then be administered to assess the participant's memories of the experiment. The Stanford Sleepiness Scale will also be re-administered. The participant will then be discharged home with the Dreem device to assess structure of sleep on the night of the study. They will also receive standard post-sedation discharge instructions and be accompanied home.

\section{Dexmedetomidine Plasma Concentrations}

To corroborate and correct the predicted plasma concentrations of dexmedetomidine, $3 \mathrm{~mL}$ venous blood samples will be collected twice during the experiment: the first immediately preceding phase IV and the second immediately preceding phase VI. Samples will be drawn from the IV not in use for infusing dexmedetomidine. Plasma dexmedetomidine levels will be measured from samples using a validated liquid chromatography-mass spectrometry assay at a specialized laboratory.

\section{Post-Sedation Sleep and MRI}

Participants will be instructed to wear the Dreem and record data during overnight sleep on the night following the sedation protocol. They will return the device the following day and complete a final sleep questionnaire and sleepiness assessment. Participants will also be scheduled for a future optional non-contrast brain MRI to facilitate source localization. The anatomical data obtained from the individual brain MRI will be used for source localization of EEG slow-waves, using Brainstorm ${ }^{46}$ (Supplemental Methods).

\section{EEG Processing and Analyses}

Processing of EEG data will be performed using customwritten MATLAB scripts and EEGLAB ${ }^{47}$ These steps will include $0.1-50 \mathrm{~Hz}$ bandpass filtering, temporal downsampling to $125 \mathrm{~Hz}$, visual rejection of bad channels, spherical spline interpolation, and re-referencing to the global average. Measures of slow-wave activity (SWA) will be derived from the power in the $0.5-4 \mathrm{~Hz}$ frequency band. Given prior studies have reported a stimulation specific increase in SWA, cumulative slow-wave activity (cSWA) will also be calculated. ${ }^{9}$ Peaks of slow-waves will be identified using automated multiscale peak detection. ${ }^{48}$ Amplitude of individual slow-waves, slope and duration of slow-wave trains will be computed. Slow-wave features and power will be compared between stimulation periods (in-phase, anti-phase) and periods with no stimulation (washout and sham). Source estimation for slow waves will be performed as described in Supplemental Methods, leveraging a non-contrast brain MRI, if taken. ${ }^{46}$ Sleep homeostasis during pre- and postsedation sleep will be assessed via changes in EEG SWA and slow-wave features between the first and last sleep cycles of the night, as well as cSWA for the entire night. ${ }^{9,13,49}$

\section{Statistical Analysis}

A linear mixed effects model will be used to compare primary outcome measures across the three conditions. The model will include time and condition (sham, in-phase, and anti-phase stimulation) as fixed effects and account for age 
and sex. Dependent variables will include slow wave features and power as described above. Paired t-tests or $U$-tests along with appropriate confidence intervals will be used to compare measures of sleep homeostasis between pre- and postsedation sleep (depending on normality of measures). Permutation tests will allow comparisons of source estimation models. Logistic regression modelling of responsiveness to thermal stimulation will be used to compare arousal thresholds during in-phase vs anti-phase vs sham stimulation.

A convenience sample size of 20 participants will be used for this pilot study. Based on prior studies involving sedation with sevoflurane, ${ }^{50}$ ketamine, ${ }^{51}$ and isoflurane, ${ }^{52}$ we expect an attrition rate of 25\%, leaving 14-15 participants with interpretable data. Considering that CLASS-D is the first study to investigate closed-loop acoustic stimulation during sedation, the effect sizes for our primary and secondary outcomes are currently unknown. Previous studies investigating closed-loop acoustic stimulation during sleep have used sample sizes of 11-28 participants to detect changes in SWA of $10-40 \%{ }^{9-13,53}$ Based on this literature, we conservatively expect CLASS-D to be powered to detect a change in slow wave features and power of $15 \%$, with an alpha of 0.05 and beta of 0.8 . Of note, some participants may entirely skip phase II of the experiment, thus making it difficult to estimate how many participants will have interpretable "Light CLASS" data. Assuming that participants will exhibit an average respiratory rate of 12 breaths per minute, which is at the lower end of a normal range, each participant who goes through phase II of the experiment will complete 72 behavioral trials (ie, 12 opportunities to squeeze the dynamometer per minute, for 6 minutes). Pre-specifying alpha to 0.05 and beta to 0.8 , power analysis for within-subject comparisons shows that 69 trials per condition are needed to detect a 0.25 effect size. ${ }^{54}$ This effect size is consistent with a recent meta-analysis quantifying the effect of acoustic slow wave enhancement on memory consolidation, ${ }^{55}$ although admittedly this represents a distinct downstream effect of slow wave enhancement.

\section{Conclusion}

This investigation will elucidate 1) the potential of exogenous stimulation to potentiate EEG slow waves during sedation; 2) the physiologic significance of this intervention; and 3) the connection between EEG slow-waves observed during sleep and sedation.

\section{Abbreviations}

ASA, American Society of Anesthesiologists; CLAS, Closedloop acoustic stimulation; CLASS, Closed-loop acoustic stimulation during sedation; CLASS-D, Closed-Loop Acoustic Stimulation during Sedation with Dexmedetomidine; DEX, Dexmedetomidine; EEG, Electroencephalogram; GABA, Gamma aminobutyric acid; HPT, Heat pain threshold; HPTT, Heat pain tolerance threshold; IV, Intravenous; N3, Non-rapid eye movement sleep Stage 3; NREM, Non-rapid Eye Movement; QST, quantitative sensory testing; SWA, slow-wave activity; TCI, targetcontrolled infusion.

\section{Data Sharing Statement}

Dissemination plans include presentations at scientific conferences and scientific publications. Acquired data will be made available upon reasonable request and results will be made available to the public via ClincialTrials.gov.

\section{Ethics Approval and Informed Consent}

This protocol includes all the elements elaborated in the SPIRIT (Standard Protocol Items: Recommendations for Interventional Trials) checklist. ${ }^{56,57}$ It was not appropriate to involve patients or the public in the design, or conduct, or reporting, or dissemination plans of our research. The study was approved by the HRPO at Washington University in St. Louis (IRB: 2019007086). The principal investigator (PI) or collaborators will obtain written informed consent prior to any study procedures. This study has been registered publicly on the ClincalTrials. gov website (NCT04206059) and will be conducted in accordance with the Declaration of Helsinki.

\section{Acknowledgments}

The authors thank Courtney Chan for assistance with developing figures, Karen Frey for guidance on QST, Michael Prerau for guidance on the behavioral task, Uwe Christians with assistance on dexmedetomidine quantitation, and Nir Grossman and David Wang for guidance on ecHT.

\section{Author Contributions}

All authors made a significant contribution to the work reported, whether that is in the conception, study design, execution, acquisition of data, analysis and interpretation, or in all these areas; took part in drafting, revising or critically reviewing the article; gave final approval of the version to be published; have agreed on the journal to 
which the article has been submitted; and agree to be accountable for all aspects of the work.

\section{Funding}

Funding from the Washington University Department of Anesthesiology and the National Institutes of Health R01AG057901 (BJAP) supported the production of this manuscript. Dr. Emery Brown was supported partially by NIH grants P01-GM118629 and R01 MH117063.

\section{Disclosure}

Dr. Simon Haroutounian reports grants from Disarm Therapeutics, personal fees from Medoc Ltd and Rafa Laboratories, outside the submitted work. Dr. Emery $\mathrm{N}$ Brown was involved in the start-up company Neuradia that is investigating drugs to induce wake up from anesthesia and a start-up company that is developing systems for control of physiological states during anesthesia. Dr. Emery N Brown has pending patents US20200187853A1 and US20190374158A1, and receives royalties from Masimo for pending patent US16373498 and issued patent US10299720. Dr. Christian S Guay, Dr. Emery N Brown and Dr. Ben Julian A Palanca report pending patent US 17/128,845. The authors report no other conflicts of interest in this work.

\section{References}

1. Prerau MJ, Brown RE, Bianchi MT, Ellenbogen JM, Purdon PL. Sleep neurophysiological dynamics through the lens of multitaper spectral analysis. Physiology (Bethesda). 2017;32(1):60-92. doi:10.1152/ physiol.00062.2015

2. Leger D, Debellemaniere E, Rabat A, Bayon V, Benchenane K, Chennaoui M. Slow-wave sleep: from the cell to the clinic. Sleep Med Rev. 2018;41:113-132.

3. Neske GT. The slow oscillation in cortical and thalamic networks: mechanisms and functions. Front Neural Circuits. 2015;9:88. doi:10.3389/fncir.2015.00088

4. Greene RW, Frank MG. Slow wave activity during sleep: functional and therapeutic implications. Neuroscientist. 2010;16(6):618-633. doi: $10.1177 / 1073858410377064$

5. Borbely AA. A two process model of sleep regulation. Hum Neurobiol. 1982;1(3):195-204.

6. Marshall L, Helgadottir H, Molle M, Born J. Boosting slow oscillations during sleep potentiates memory. Nature. 2006;444(71 19):610-613. doi:10.1038/nature05278

7. Massimini M, Ferrarelli F, Esser SK, et al. Triggering sleep slow waves by transcranial magnetic stimulation. Proc Natl Acad Sci U S A. 2007;104(20):8496-8501. doi:10.1073/pnas.0702495104

8. Ngo HV, Martinetz T, Born J, Molle M. Auditory closed-loop stimulation of the sleep slow oscillation enhances memory. Neuron. 2013;78 (3):545-553. doi:10.1016/j.neuron.2013.03.006

9. Garcia-Molina G, Tsoneva T, Jasko J, et al. Closed-loop system to enhance slow-wave activity. J Neural Eng. 2018;15(6):066018. doi:10.1088/1741-2552/aae18f
10. Besedovsky L, Ngo HV, Dimitrov S, Gassenmaier C, Lehmann R, Born J. Auditory closed-loop stimulation of EEG slow oscillations strengthens sleep and signs of its immune-supportive function. Nat Commun. 2017;8(1):1984. doi:10.1038/s41467-017-02170-3

11. Leminen MM, Virkkala J, Saure E, et al. Enhanced memory consolidation via automatic sound stimulation during non-REM sleep. Sleep. 2017;40(3). doi:10.1093/sleep/zsx003.

12. Papalambros NA, Santostasi G, Malkani RG, et al. Acoustic enhancement of sleep slow oscillations and concomitant memory improvement in older adults. Front Hum Neurosci. 2017;11:109. doi:10.3389/ fnhum.2017.00109

13. Grimaldi D, Papalambros NA, Reid KJ, et al. Strengthening sleep-autonomic interaction via acoustic enhancement of slow oscillations. Sleep. 2019;42. Doi:10.1093/sleep/zsz036

14. Schreglmann SR, Wang D, Peach RL, et al. Non-invasive suppression of essential tremor via phase-locked disruption of its temporal coherence. Nat Commun. 2021;12(1):363. doi:10.1038/s41467-02020581-7

15. Debellemaniere E, Chambon S, Pinaud C, et al. Performance of an ambulatory dry-EEG device for auditory closed-loop stimulation of sleep slow oscillations in the home environment. Front Hum Neurosci. 2018;12:88. doi:10.3389/fnhum.2018.00088

16. Bellesi M, Riedner BA, Garcia-Molina GN, Cirelli C, Tononi G. Enhancement of sleep slow waves: underlying mechanisms and practical consequences. Front Syst Neurosci. 2014;8:208. doi:10.3389/ fnsys.2014.00208

17. Akeju O, Hobbs LE, Gao L, et al. Dexmedetomidine promotes biomimetic non-rapid eye movement stage 3 sleep in humans: a pilot study. Clin Neurophysiol. 2018;129(1):69-78. doi:10.1016/j. clinph.2017.10.005

18. Brown EN, Lydic R, Schiff ND, Schwartz RS. General anesthesia, sleep, and coma. $N$ Engl J Med. 2010;363(27):2638-2650. doi:10.1056/NEJMra0808281

19. Purdon PL, Sampson A, Pavone KJ, Brown EN. Clinical electroencephalography for anesthesiologists: part I: background and basic signatures. Anesthesiology. 2015;123(4):937-960. doi:10.1097/ALN. 0000000000000841

20. Akeju O, Kim SE, Vazquez R, et al. Spatiotemporal dynamics of dexmedetomidine-induced electroencephalogram oscillations. PLoS One. 2016;11(10):e0163431. doi:10.1371/journal.pone.0163431

21. Xi C, Sun S, Pan C, Ji F, Cui X, Li T. Different effects of propofol and dexmedetomidine sedation on electroencephalogram patterns: wakefulness, moderate sedation, deep sedation and recovery. PLoS One. 2018;13(6):e0199120. doi:10.1371/journal. pone. 0199120

22. Ebert TJ, Hall JE, Barney JA, Uhrich TD, Colinco MD. The effects of increasing plasma concentrations of dexmedetomidine in humans. Anesthesiology. 2000;93(2):382-394. doi:10.1097/00000542-200008 000-00016

23. Sleigh JW, Vacas S, Flexman AM, Talke PO. Electroencephalographic arousal patterns under dexmedetomidine sedation. Anesth Analg. 2018;127(4):951-959. doi:10.1213/ANE.000 0000000003590

24. Scheinin A, Kallionpaa RE, Li D, et al. Differentiating drug-related and state-related effects of dexmedetomidine and propofol on the electroencephalogram. Anesthesiology. 2018;129(1):22-36. doi:10.10 97/ALN.0000000000002192

25. Guldenmund P, Vanhaudenhuyse A, Sanders RD, et al. Brain functional connectivity differentiates dexmedetomidine from propofol and natural sleep. Br J Anaesth. 2017;119(4):674-684. doi:10.1093/bja/ aex 257

26. Ni Mhuircheartaigh R, Warnaby C, Rogers R, Jbabdi S, Tracey I. Slow-wave activity saturation and thalamocortical isolation during propofol anesthesia in humans. Sci Transl Med. 2013;5(208):20 8ra148. doi:10.1126/scitranslmed.3006007 
27. Schmid W, Marhofer P, Opfermann P, et al. Brainwave entrainment to minimise sedative drug doses in paediatric surgery: a randomised controlled trial. Br J Anaesth. 2020;125:330-335. doi:10.1016/j. bja.2020.05.050

28. Connor CW, Forensic A. Disassembly of the BIS monitor. Anesth Analg. 2020;131(6):1923-1933. doi:10.1213/ANE.0000000000005220

29. Sigl JC, Chamoun NG. An introduction to bispectral analysis for the electroencephalogram. J Clin Monit. 1994;10(6):392-404. doi:10.10 07/BF01618421

30. Rolke R, Baron R, Maier C, et al. Quantitative sensory testing in the German Research Network on Neuropathic Pain (DFNS): standardized protocol and reference values. Pain. 2006;123(3):231-243. doi:10.1016/j.pain.2006.01.041

31. Arnal PJ, Thorey V, Ballard ME, et al. The Dreem headband as an alternative to polysomnography for EEG signal acquisition and sleep staging. BioRxiv. 2019;662734.

32. Andrillon T, Solelhac G, Bouchequet P, et al. Revisiting the value of polysomnographic data in insomnia: more than meets the eye. Sleep Med. 2020;66:184-200. doi:10.1016/j.sleep.2019.12.002

33. Hannivoort LN, Eleveld DJ, Proost JH, et al. Development of an optimized pharmacokinetic model of dexmedetomidine using target-controlled infusion in healthy volunteers. Anesthesiology. 2015;123(2):357-367. doi:10.1097/ALN.0000000000000740

34. Colin PJ, Hannivoort LN, Eleveld DJ, et al. Dexmedetomidine pharmacodynamics in healthy volunteers: 2 . Haemodynamic profile. $\mathrm{Br}$ $J$ Anaesth. 2017;119(2):211-220. doi:10.1093/bja/aex086

35. Colin PJ, Hannivoort LN, Eleveld DJ, et al. Dexmedetomidine pharmacokinetic-pharmacodynamic modelling in healthy volunteers: 1. Influence of arousal on bispectral index and sedation. Br J Anaesth. 2017;119(2):200-210. doi:10.1093/bja/aex085

36. Weerink MAS, Struys M, Hannivoort LN, Barends CRM, Absalom AR, Colin P. Clinical pharmacokinetics and pharmacodynamics of dexmedetomidine. Clin Pharmacokinet. 2017;56 (8):893-913. doi:10.1007/s40262-017-0507-7

37. Hoddes E, Zarcone V, Smythe H, Phillips R, Dement WC. Quantification of sleepiness: a new approach. Psychophysiology. 1973;10(4):431-436. doi:10.1111/j.1469-8986.1973.tb00801.x

38. Pandya AN, Majid SZ, Desai MS. The origins, evolution, and spread of anesthesia monitoring standards: from Boston to across the world. Anesth Analg. 2020. doi:10.1213/ANE.0000000000005021

39. Prerau MJ, Hartnack KE, Obregon-Henao G, et al. Tracking the sleep onset process: an empirical model of behavioral and physiological dynamics. PLoS Comput Biol. 2014;10(10):e1003866. doi:10.1371/ journal.pcbi.1003866

40. Guay CS, Plourde G. Handgrip dynamometry for continuous assessment of volitional control during induction of anesthesia: a prospective observational study. Can J Anaesth. 2019;66(1): 48-56. doi:10.1007/s12630-018-1224-x

41. Purdon PL, Pierce ET, Mukamel EA, et al. Electroencephalogram signatures of loss and recovery of consciousness from propofol. Proc Natl Acad Sci U S A. 2013;110(12):E1142-1151. doi:10.1073/pnas.12 21180110
42. Purdon PL, Colvin CA, Brown EN EEG for anesthesia. International Anesthesia Research Society; 2019. Available from: http://eegfora nesthesia.iars.org/. Accessed February 19, 2021.

43. Molle M, Born J. Slow oscillations orchestrating fast oscillations and memory consolidation. Prog Brain Res. 2011;193:93-110.

44. Talke P, Anderson BJ. Pharmacokinetics and pharmacodynamics of dexmedetomidine-induced vasoconstriction in healthy volunteers. $\mathrm{Br}$ J Clin Pharmacol. 2018;84(6):1364-1372. doi:10.1111/bcp.13571

45. Aldrete JA. The post-anesthesia recovery score revisited. J Clin Anesth. 1995;7(1):89-91. doi:10.1016/0952-8180(94)00001-K

46. Tadel F, Baillet S, Mosher JC, Pantazis D, Leahy RM. Brainstorm: a user-friendly application for MEG/EEG analysis. Comput Intell Neurosci. 2011;2011:879716. doi:10.1155/2011/879716

47. Delorme A, Makeig S. EEGLAB: an open source toolbox for analysis of single-trial EEG dynamics including independent component analysis. J Neurosci Methods. 2004;134(1):9-21. doi:10.1016/j. jneumeth.2003.10.009

48. Scholkmann F, Bens J, Wolf M. An efficient algorithm for automatic peak detection in noisy periodic and quasi-periodic signals. Algorithms. 2012;5:588-603. doi:10.3390/a5040588

49. Riedner BA, Vyazovskiy VV, Huber R, et al. Sleep homeostasis and cortical synchronization: III. A high-density EEG study of sleep slow waves in humans. Sleep. 2007;30(12):1643-1657. doi:10.1093/sleep/ 30.12.1643

50. Palanca BJ, Mitra A, Larson-Prior L, Snyder AZ, Avidan MS, Raichle ME. Resting-state functional magnetic resonance imaging correlates of sevoflurane-induced unconsciousness. Anesthesiology. 2015;123:346-356. doi:10.1097/ALN.0000000000000731

51. Palanca BJA, Maybrier HR, Mickle AM, et al. Cognitive and neurophysiological recovery following electroconvulsive therapy: a study protocol. Front Psychiatry. 2018;9:171. doi:10.3389/fpsyt.2018.00 171

52. Maier KL, McKinstry-Wu AR, Palanca BJ, et al. Protocol for the reconstructing human consciousness and cognition study. Front Hum Neurosci. 2017. doi:10.3389/fnhum.2017.00284

53. Ong JL, Lo JC, Chee NI, et al. Effects of phase-locked acoustic stimulation during a nap on EEG spectra and declarative memory consolidation. Sleep Med. 2016;20:88-97. doi:10.1016/j.sleep.20 15.10 .016

54. Casagrande JT, Pike MC, Smith PG. An improved approximate formula for calculating sample sizes for comparing two binomial distributions. Biometrics. 1978;34(3):483-486. doi:10.2307/2530613

55. Wunderlin M, Zust MA, Hertenstein E, et al. Modulating overnight memory consolidation by acoustic stimulation during slow wave sleep - a systematic review and meta-analysis. Sleep. 2021. doi:10.1093/sleep/zsaa296

56. Chan AW, Tetzlaff JM, Gotzsche PC, et al. SPIRIT 2013 explanation and elaboration: guidance for protocols of clinical trials. $B M J$. 2013;346:e7586

57. Chan AW, Tetzlaff JM, Altman DG, et al. SPIRIT 2013 statement: defining standard protocol items for clinical trials. Ann Intern Med. 2013;158 (3):200-207. doi:10.7326/0003-4819-158-3-201302050-00583

\section{Publish your work in this journal}

Nature and Science of Sleep is an international, peer-reviewed, open access journal covering all aspects of sleep science and sleep medicine, including the neurophysiology and functions of sleep, the genetics of sleep, sleep and society, biological rhythms, dreaming, sleep disorders and therapy, and strategies to optimize healthy sleep.
The manuscript management system is completely online and includes a very quick and fair peer-review system, which is all easy to use. Visit http://www.dovepress.com/testimonials.php to read real quotes from published authors. 